\title{
Understanding the Utility of Digital Flight Assistants: A Preliminary Analysis
}

\author{
Sarah Gosper \\ sgosper@student.unimelb.edu.au \\ University of Melbourne \\ Melbourne, Australia \\ Fraser Allison \\ fraser.allison@unimelb.edu.au \\ University of Melbourne \\ Melbourne, Australia
}

\author{
Johanne R. Trippas \\ johanne.trippas@unimelb.edu.au \\ University of Melbourne \\ Melbourne, Australia \\ Cynthia Sear \\ cynthia.sear@unimelb.edu.au \\ University of Melbourne \\ Melbourne, Australia \\ Fabio Mattioli \\ fabio.mattioli@unimelb.edu.au \\ University of Melbourne \\ Melbourne, Australia
}

\author{
Harriette Richards \\ harriette.richards@unimelb.edu.au \\ University of Melbourne \\ Melbourne, Australia \\ Sara Khorasani \\ skhorasani@student.unimelb.edu.au \\ University of Melbourne \\ Melbourne, Australia
}

\begin{abstract}
Interactions with intelligent systems have become common in domestic and professional life. However, little is known about how pilots, who already work in a highly automated environment, envisage using intelligent systems in their work environment. This preliminary analysis investigates pilots' needs and wants for digital flight assistants (DFAs) through an interview study. We show that the adoption of DFAs may be hindered by pre-existing concerns, such as inadequate automatic speech recognition, linked to past experiences with digital assistants. Furthermore, we identify important contextual and environmental factors that will need to be accounted for in the design of DFAs such as "cross cockpit" relationships, noisy environments, or pilot's cognitive workload.
\end{abstract}

\section{CCS CONCEPTS}

- Hardware $\rightarrow$ Emerging interfaces; $\bullet$ Human-centered computing $\rightarrow$ Natural language interfaces.

\section{KEYWORDS}

conversational systems, cockpits, autonomy

\section{ACM Reference Format:}

Sarah Gosper, Johanne R. Trippas, Harriette Richards, Fraser Allison, Cynthia Sear, Sara Khorasani, and Fabio Mattioli. 2021. Understanding the Utility of Digital Flight Assistants: A Preliminary Analysis. In CUI 2021 - 3rd Conference on Conversational User Interfaces (CUI '21), July 27-29, 2021, Bilbao (online), AA, Spain. ACM, New York, NY, USA, 5 pages. https: //doi.org/10.1145/3469595.3469627

Permission to make digital or hard copies of all or part of this work for personal or classroom use is granted without fee provided that copies are not made or distributed for profit or commercial advantage and that copies bear this notice and the full citation on the first page. Copyrights for components of this work owned by others than the author(s) must be honored. Abstracting with credit is permitted. To copy otherwise, or republish, to post on servers or to redistribute to lists, requires prior specific permission and/or a fee. Request permissions from permissions@acm.org.

CUI '21, fuly 27-29, 2021, Bilbao (online), AA, Spain

(c) 2021 Copyright held by the owner/author(s). Publication rights licensed to ACM ACM ISBN 978-1-4503-8998-3/21/07 . \$15.00

https://doi.org/10.1145/3469595.3469627

\section{INTRODUCTION}

Digital assistants such as Apple's Siri, Google Home, or Microsoft's Cortana are increasing in popularity [10]. These intelligent systems are slowly but steadily penetrating our home environment, enabling us to access information while cooking, switch on our lights when we wake up, or ask for music. More recently, research has investigated how digital assistants can be used to support people engaged in work-related tasks such as collaborations in the workplace $[16,23]$. There has been a growing interest in the development of workplace-oriented digital assistants, such as Alexa for Business ${ }^{1}$ and Cortana Skills Kit for Enterprise ${ }^{2}$, but the uptake of these and similar systems remains limited so far. Currently available digital assistants often have limited capabilities, such as dictation and simple reminders, and are referred to as "passive" or "command-and-control" systems [17, 22]. To date, the main target users for these systems have been white-collar workers in traditional office environments rather than workers in more varied and complex environments such as aeroplane pilots.

In aviation, there are several potential advantages to incorporating intelligent systems into the cockpit in the form of digital flight assistants (DFAs). Recent papers have noted that the complexity of technical information in the aerospace domain makes conversational systems potentially useful in the cockpit, but also particularly complex to design and evaluate $[1,2]$. DFAs could optimise route planning, alleviate the pilot's cognitive burden and provide detailed warnings of errors detected by sensors throughout the aircraft. DFAs could facilitate communications with air traffic control, a domain in which automatic speech recognition systems have already been introduced [12].

Automated systems, which are often implemented using heuristics, are already highly embedded in the aviation industry. These systems require no human intervention but are based on a fixed set of rules and are therefore reasonably predictable; this is in contrast to autonomous systems. Although aviation is a risk-averse

\footnotetext{
${ }^{1}$ https://aws.amazon.com/alexaforbusiness/

${ }^{2}$ https://blogs.microsoft.com/ai/cortana-for-enterprise/
} 
and safety-critical sector, interest in autonomous systems within the cockpit has been rising, alongside digital tools such as the electronic flight bag [8]. Autonomy in aviation has been predicted to be crucial due to the increase of air traffic ${ }^{3}$, and some forms of intelligent systems are likely to be crucial to manage this rising volume of flights $[14,19]$. Several studies have investigated people's willingness to fly on autonomous aeroplanes, and its relationship to demographic and contextual variables such as gender, nationality and weather [18, 24]. A predictive model developed by Rice et al. [21] suggested that willingness to fly on an autonomous aeroplane was positively associated with familiarity with the technology, and negatively associated with caution towards new technologies. In this context, DFAs could represent a partial step towards more autonomous flights while retaining human pilots to preserve passengers' trust in aviation. However, in contrast to passenger attitudes, little is known about how pilots perceive the utility of DFAs. We aim to address this gap.

The remainder of the paper is structured as follows. Section 2 describes the survey methodology. Section 3 describes the preliminary results of the qualitative analysis. We discuss the results and their implications and conclude in Section 4 with a summary of our findings and potential avenues for future work.

\section{METHODS}

\subsection{Approach}

This paper describes the first phase (Oct-Dec 2020) of a study aimed at investigating aeroplane pilots' views of automation, specifically conversational systems in the cockpit. Currently, several avionics companies are studying the introduction of digital flight assistants designed to support or even replace (one of the) pilots. To understand how such a technology could be made more effective, we researched:

(1) how pilots utilise and experience existing personal digital assistants (e.g., Alexa and Siri), and

(2) the role that pilots would want digital assistants to take in the cockpit.

We focused on asking participants about inter-relational dimensions of flying and conversational interfaces, including how co-pilots relate to one another in high-stress and low-stress phases of flight. The investigation included asking participants about their trust or distrust of digital assistants, how the voice of a digital assistant might intervene in the already noisy cockpit environment, and how portable or adaptable digital assistants could be used in the cockpit. We consider our findings in relation to existing research about automation in the cockpit to draw preliminary conclusions as to the utility of voice-activated digital flight assistants.

\subsection{Procedure}

Ethics approval was obtained from the university's human research ethics committee ${ }^{4}$. The study was classified as low risk. The interviewers explained their role and the aim of the study. Participants were invited to provide consent and were sent a list of possible

\footnotetext{
${ }^{3}$ https://www.iata.org/contentassets/bccae1c5a24e43759607a5fd8f44770b/vision2050.pdf

${ }^{4}$ Pseudonyms are used in this paper to preserve anonymity.
}

themes of discussion. Semi-structured interviews were conducted online via Zoom ${ }^{5}$ due to the international scope of the research, global lockdowns, and working-from-home restrictions necessitated by the COVID-19 pandemic. Interviews were facilitated by two researchers and were each at least one hour long. In some instances, participants chose to share their screen with the interviewers to demonstrate aspects of the digital interfaces and databases that they use when flying. All interviews were recorded and transcribed by the researchers, with participants assigned pseudonyms.

\subsection{Participants}

In the first stage, we interviewed fifteen participants in thirteen online interviews (one interview was conducted as a group, with three pilots participating). Recruitment was conducted through personal contacts from the authors and snowball sampling. Fourteen of the participants were male and one was female. This gender bias reflects that of the aviation industry ${ }^{6}$, in which only $8 \%$ of pilots are female. Participants were not requested to provide their age, but they differed broadly in their aviation experience. Seven participants were Captains, with the most experienced pilot having flown for over 37 years. Four were First Officers, with the youngest having flown for just six years. The remaining four pilots were trainers/trainees at flight schools. The majority $(>90 \%)$ of participants used English as their first language, with eight participants based in Australia, four in New Zealand, and three elsewhere. Besides the four trainers/trainees, all participants were employed as either international or domestic commercial pilots flying multi-crew operations. The majority $(>70 \%)$ of participants had been grounded due to flight restrictions caused by the COVID-19 pandemic.

\subsection{Qualitative Analysis}

Data collected from the interviews were analysed collaboratively by the team members. Two authors coded the interview transcripts thematically using an inductive coding process [6]. From this data set, the authors collectively identified key insights regarding the pilots' perceptions of DFA utility in the cockpit.

\section{RESULTS}

In this section, we first describe how the pilots discussed their experiences with existing personal digital assistants. We then present the initial results for how the pilots thought about the introduction of digital assistants into the cockpit.

\subsection{Technology Adoption and Curiosity}

Overall, the participants had a positive attitude towards the usage of technology in an everyday and professional setting. Participants mentioned that they were on top of the latest developments around intelligent systems and that using these systems was already embedded in their everyday routine. Jake shared, "I use Siri every night to set alarms, to set reminders. I use it for alarms, reminders and stuff in my calendar. Checking the weather." Many participants expressed interest in keeping up-to-date with new technologies such as augmented and virtual reality, drones and smart home integration.

\footnotetext{
$\overline{5 \text { https://zoom.us/ }}$

${ }^{6}$ https://www.faa.gov/data_research/aviation_data_statistics/civil_airmen_statistics/
} 
Several pilots emphasised that they were particularly interested in "practical" technology, as Peter stated:

"I do embrace technology, because of my electrical background and stuff, I really love technology that's useful and, I'm not being rude, I'm not really interested in Instagram. I've got a Facebook account, but you know, I don't really look at anything like that. I Bluetooth my phone to a speaker in home and stuff like that."

Many of the pilots expressed a willingness to think beyond existing technologies to optimise their day-to-day activities. Jake described: "What I wanted to do was use a Raspberry Pi and an IR transmitter to somehow track my car and so when I got within 100 metres of my garage, the door automatically opens, so as I pull up it's already open and I can just drive in, as opposed to having to click a button." This suggests an interest in tailoring digital systems to their individual needs and preferences, rather than being satisfied with the design of pre-packaged products.

The airline pilots also expressed positive views of automated technologies in their work environment, the cockpit. Most suggested that technologies such as autopilots, auto-thrust systems and assisted landing helped to significantly reduce their workload in flight.

Despite this positive attitude towards automated technologies in general, the participants expressed reservations about voice interaction with a DFA in the cockpit. They questioned the purpose of adding a voice-based tool if it would only replicate functionality that was already served by non-voice-based systems (such as priority-based electronic checklists). In particular, participants were not sure what value a voice interface could add besides the "audification" of existing data. Ramona mentioned, "It is calculating how far you can go before you can do it, so this stuff is already happening [with written down data]. Would there be any benefit for it to be audible, would be my question." Participants were particularly concerned that they would miss a vocal notification from the DFA during flight, due to the many different sounds and background noises in the cockpit, which would add to the stress of flying.

\subsection{Hierarchy on the Workfloor}

Many of the airline pilots' comments concerned the ways in which a DFA would affect their working relationships (particularly between co-pilots and with the flight crew) or the cockpit environment. Some participants mentioned concerns around the privacy of data generated by the DFA, suggesting that it might become particularly contentious in companies where pilots felt less supported.

Because civil aviation is a hierarchical environment in which interactions are highly scripted, any slight adaption can change relationships dramatically. Participants mentioned that a DFA could introduce friction or challenge existing lines of authority in the cockpit because it may be seen as an extra entity or virtual "person", in the role of a backseat driver. Muhammad said that it would affect the normal relationship dynamics between pilots, because "some people would see it as a third set of eyes that are judging your actions". Some participants mentioned that a DFA could undermine a captain's authority if he or she proved to be less adept with the new technology than their first officer. Mike described the scenario:
"You've got the authority gradient. The captain is the more senior and [then] the first officer and then potentially others. I could certainly see a situation where Captain was trying to interact with the voice assistant and it wasn't working, the first officer then interacts with the voice system, gets it to work, Captain then feels inferior and loses the authority gradient."

Pilots mentioned the importance of adaptiveness for a DFA, in the sense that it could either be pro-active or reactive to prompts by the pilots, and thus included as part of the team. Different kinds of collaboration modes were mentioned as being helpful, such as multi-party collaboration as opposed to a one-on-one user-agent interaction. Somewhat contrary to this, however, was a concern that a DFA could take on too prominent a role in the cockpit and thereby disrupt the learning opportunities that are generated by pilots' collaborative approach to rectifying mistakes.

Concerns also extended to the impact of a DFA on the work environment. Participants mentioned that cockpits are already loud due to the aircraft's mechanical functions, external communications (e.g., with air traffic controllers) and internal communications (e.g., with flight crew). Frequent verbal safety checks, updates and alerts create a loud and busy workplace atmosphere. Hence, many pilots were concerned about the addition of a DFA as another voice in the mix, and one that would not be sensitive to context. Existing aircraft are designed to produce voice alerts in extreme situations, such as when a plane is about the crash; concerns about how a DFA would work and know when to speak in these crucial but rare circumstances were open questions [20].

\subsection{Digital Flight Assistants' Perceived Utility}

Participants had an overall positive outlook on the prospects for DFAs to be implemented in the future. However, they pointed out that DFAs would need to meet higher standards of reliability and usefulness than the commercial digital assistants with which they had previous experience. Pilots noted that commercial digital assistants, such as Siri, frequently make mistakes; do not have sufficiently robust systems for sourcing information or allowing users to double-check the information provided (a lack of transparency also noted by [5], which our pilots referred to as the "bullshit in / bullshit out" factor); and cannot yet compare to human decision-making. These considerations made the airline pilots doubt that DFAs could meet the industry's regulations for system failures. Furthermore, many participants were sceptical that a DFA would understand all the different scenarios when flying an aircraft, as Sam stated:

"We could probably get there, but at the end of the day, there are so many scenarios that sometimes don't require any technical knowledge but still need that human, I guess, perspective on it, kind of like medical [emergencies] or unruly passengers."

Nevertheless, participants anticipated three particular advantages to the future presence of DFAs in the cockpit:

- Increased safety in time-critical scenarios, such as retrieving information about diversion options due to bad weather ahead of time. 
- Pilot monitoring, so that if a pilot made a mistake or appeared to be in an impaired state, an "emotionally intelligent" DFA could alert them.

- Integration of existing distributed systems into one centralised interface.

The majority of participants stated that a DFA would be most useful in single-pilot flights, where it could provide weather updates, help to reduce the pilot's cognitive workload [15] and potentially mitigate boredom and fatigue [3]. Jake said:

"I can see the biggest use of a digital flight assistant would be in a single pilot environment. Like driving aids in cars that like detect when you're getting sleepy, if you hit the rumble strip too many times, it comes up and says hey, we feel like you're getting less alert."

\section{DISCUSSION AND CONCLUSION}

This study aimed to explore pilots' relationships with and attitudes towards conversational digital assistants, both in their current commercial forms and as hypothetical near-future technologies that could be introduced into the cockpit. Professional airline pilots demonstrated a significant level of personal and professional curiosity towards new automated technologies. However, they indicated several ways in which DFAs would need to be tailored to the aviation context before they would be fit for purpose in the cockpit. Unlike general consumers, airline pilots operate in a highly automated environment. As a result, our participants saw some practical uses of DFAs as redundant. For instance, they suggested that they did not need to "audiofy" existing information. However, they could think of use-cases beyond the command-and-control interaction paradigm where DFA would have practical applications. Furthermore, adding an extra speech-only system in an already noisy environment could potentially be a safety concern, future research could investigate the multi-modality of DFAs [9].

Pilots expressed particularly high willingness to fly with DFAs in the setting of a single-pilot operation, which is similar to findings by Battiste et al. [4]. Furthermore, the pilots highlighted that, to be successful, a DFA should be able not only to provide a practical support, but also to read the relational context of the cockpit. Otherwise, adding an extra "entity" into an already highly formulated workplace, within the deeply interconnected socio-technical system of aviation, could be disruptive to existing work practices. This finding is in line with prior work by Huang [13], which stresses how autonomous flight systems cannot be taken out of their context and environmental complexity. Despite the participant's concerns, potentially, a DFA could improve work relationships [7]. For example, the DFA could be a mentor or teacher during flights or provide entertainment during long flights, helping with the "cross cockpit" hierarchy. Overall, the preliminary results of this research suggest that conversational digital assistants intended for professional use in aviation will need to be adapted to the specific cyber-physicalsocial activities that they aim to enhance [23].

\subsection{Limitations and Future Work}

This initial analysis of pilots' relationship with digital assistants was performed to explore how pilots, who work in an already highly automated environment, use such systems in their daily lives and see their future usage in the cockpit. The fact that all but one of the participants were men, although reflecting the gender imbalance among pilots as a profession, is a limitation of the research. As future work, we will continue interviewing pilots to capture the views of more women, extend the analysis with a rigorous thematic analysis approach [6,11], and include further consideration of pilots' backgrounds in the analysis. The findings will be developed to inform future digital flight assistant designs.

\section{ACKNOWLEDGMENTS}

We would like to thank all the participants who took part in our study. We thank the anonymous reviewers for their useful suggestions. This work is supported by the Center for Artificial Intelligence and Digital Ethics seed funding.

\section{REFERENCES}

[1] Alexandre Arnold, Gérard Dupont, Catherine Kobus, François Lancelot, and Ying-Hsang Liu. 2020. Perceived Usefulness of Conversational Agents Predicts Search Performance in Aerospace Domain. In Proceedings of the 2nd Conference on Conversational User Interfaces. Article 57, 3 pages.

[2] Alexandre Arnold, Catherine Kobus, François Lancelot, Pooja Narayan, and Gérard Dupont. [n.d.]. Conversational agent for aerospace question answering A position paper. In Proceedings of the 1st Workshop on Conversational Interaction Systems (WCIS at SIGIR) (Paris, 2019).

[3] Randall E Bailey, Lynda J Kramer, Kellie D Kennedy, Chad L Stephens, and Timothy J Etherington. 2017. An assessment of reduced crew and single pilot operations in commercial transport aircraft operations. In 2017 IEEE/AIAA 36th Digital Avionics Systems Conference (DASC). IEEE, 1-15.

[4] Vernol Battiste, Joel Lachter, Summer Brandt, Armando Alvarez, Thomas Z Strybel, and Kim-Phuong L Vu. 2018. Human-Automation teaming: Lessons learned and future directions. In International Conference on Human Interface and the Management of Information. Springer, 479-493.

[5] Adam Bogg, Stewart Birrell, Michael A Bromfield, and Andrew M Parkes. 2020. Can we talk? How a talking agent can improve human autonomy team performance. Theoretical Issues in Ergonomics Science (2020), 1-22.

[6] Virginia Braun and Victoria Clarke. 2013. Successful Qualitative Research: A Practical Guide for Beginners. SAGE Publications.

[7] Peter A Brennan and Mike Davidson. 2019. Improving patient safety: we need to reduce hierarchy and empower junior doctors to speak up. BMF 366 (2019).

[8] Joan Cahill and Nick Mc Donald. 2006. Human computer interaction methods for electronic flight bag envisionment and design. Cognition, Technology \& Work 8,2 (2006), 113-123.

[9] Yashar Deldjoo, Johanne R. Trippas, and Hamed Zamani. 2021. Towards MultiModal Conversational Information Seeking. In Proceedings of the ACM Conference on Research and Development in Information Retrieval (SIGIR'21). 1-11.

[10] Forbes. 2018. Okay, Google, Will Voice Be The Future Of Search? Retrieved October, 2019 from https://www.forbes.com/sites/nicolemartin1/2018/11/06/ok-googlewill-voice-be-the-future-of-search/

[11] Lisa M Given. 2008. The SAGE Encyclopedia of Qualitative Research Methods. SAGE Publications.

[12] Hartmut Helmke, Heiko Ehr, Matthias Kleinert, Friedrich Faubel, and Dietrich Klakow. [n.d.]. Increased Acceptance of Controller Assistance by Automatic Speech Recognition. 1-10. https://elib.dlr.de/87600/

[13] Hui-Min Huang. 2007. Autonomy levels for unmanned systems (ALFUS) framework: safety and application issues. In Proceedings of the 2007 Workshop on Performance Metrics for Intelligent Systems. 48-53.

[14] Trevor Kistan, Alessandro Gardi, and Roberto Sabatini. 2018. Machine learning and cognitive ergonomics in air traffic management: Recent developments and considerations for certification. Aerospace 5, 4 (2018), 103.

[15] Jing Liu, Alessandro Gardi, Subramanian Ramasamy, Yixiang Lim, and Roberto Sabatini. 2016. Cognitive pilot-aircraft interface for single-pilot operations. Knowledge-based systems 112 (2016), 37-53.

[16] Moira McGregor and John C Tang. 2017. More to meetings: challenges in using speech-based technology to support meetings. In Proceedings of the 2017 ACM conference on computer supported cooperative work and social computing. 22082220 .

[17] Michael McTear, Zoraida Callejas, and David Griol. 2016. The Conversational Interface. Springer.

[18] Rian Mehta, Stephen Rice, Scott Winter, and Morgan Eudy. 2017. Perceptions of cockpit configurations: A culture and gender analysis. The International fournal of Aerospace Psychology 27, 1-2 (2017), 57-63. 
[19] Raja Parasuraman, Thomas B Sheridan, and Christopher D Wickens. 2000. A model for types and levels of human interaction with automation. IEEE Transactions on systems, man, and cybernetics-Part A: Systems and Humans 30, 3 (2000), 286-297.

[20] Claudio S. Pinhanez. 2020. HCI Research Challenges for the Next Generation of Conversational Systems. In Proceedings of the 2nd Conference on Conversational User Interfaces. Article 48, 4 pages.

[21] Stephen Rice, Scott R Winter, Rian Mehta, and Nadine K Ragbir. 2019. What factors predict the type of person who is willing to fly in an autonomous commercial airplane? Journal of Air Transport Management 75 (2019), 131-138.

[22] Johanne R. Trippas, Damiano Spina, Lawrence Cavedon, Hideo Joho, and Mark Sanderson. 2018. Informing the Design of Spoken Conversational Search. In
Proceedings of Conference on Information Interaction and Retrieval (CHIIR). 32-41. [23] Johanne R. Trippas, Damiano Spina, Falk Scholer, Ahmed Hassan Awadallah, Peter Bailey, Paul N. Bennett, Ryen W. White, Jonathan Liono, Yongli Ren, Flora D. Salim, and Mark Sanderson. 2019. Learning About Work Tasks to Inform Intelligent Assistant Design. In Proceedings of Conference on Information Interaction and Retrieval (CHIIR). 5-14.

[24] Scott R Winter, Stephen Rice, Rian Mehta, Ismael Cremer, Katie M Reid, Timothy G Rosser, and Julie C Moore. 2015. Indian and American consumer perceptions of cockpit configuration policy. fournal of air transport management 42 (2015), 226-231. 


\section{University Library}

\section{- M M N E R VA A gateway to Melbourne's research publications}

Minerva Access is the Institutional Repository of The University of Melbourne

\section{Author/s:}

Gosper, S;Trippas, J;Richards, H;Allison, F;Sear, C;Khorasani, S;Mattioli, F

Title:

Understanding the Utility of Digital Flight Assistants: A Preliminary Analysis

Date:

2021

Citation:

Gosper, S., Trippas, J., Richards, H., Allison, F., Sear, C., Khorasani, S. \& Mattioli, F. (2021). Understanding the Utility of Digital Flight Assistants: A Preliminary Analysis. 3rd Conference on Conversational User Interfaces (CUI '21), pp.1-5. ACM. https:// doi.org/10.1145/3469595.3469627.

Persistent Link:

http://hdl.handle.net/11343/274920 